05,15

\title{
Начальная восприимчивость в системах магнитных эллипсоидальных наночастиц
}

\author{
(C) А.В. Акишева, Е.С. Пьянзина \\ Уральский федеральный университет им. Б.Н. Ельцина, \\ Екатеринбург, Россия \\ E-mail: annagudkova94@gmail.com
}

Поступила в Редакцию 26 марта 2019 г.

В окончательной редакции 26 марта 2019 г.

Принята к публикации 2 апреля 2019 г.

\begin{abstract}
Проведены компьютерные эксперименты методом молекулярной динамики для исследования систем магнитных эллипсоидальных наночастиц с двумя различными ориентациями диполя внутри каждой частицы. Рассмотрены магнитные моменты, направленные параллельно или перпендикулярно главной оси вращения. Исследована начальная восприимчивость системы и проведен кластерный анализ. Полученные результаты выявили влияние ориентации магнитного момента внутри частиц одинаковой формы на самоорганизацию системы, ее микроскопические и макроскопические свойства. Для случая ориентации, параллельной главной оси, выявлено следующее: начальная восприимчивость частиц, форма которых близка к сферической, быстрее возрастает и достигает более высоких значений, чем в случае эллипсоидных частиц. Перпендикулярная ориентация магнитного момента не влияет на начальную восприимчивость так существенно, как в предыдущем случае: увеличение магнитного момента приводит к росту начальной восприимчивости, тогда как форма частицы практически не оказывает на нее влияния.
\end{abstract}

Ключевые слова: магнитные анизотропные эллипсоидальные наночастицы, моделирование методом молекулярной динамики, начальная восприимчивость, самоорганизация.

DOI: $10.21883 /$ FTT.2020.09.49780.26H

\section{1. Введение}

Наблюдаемое в последнее десятилетие интенсивное развитие технологий создания смарт-материалов обусловливает повышенный интерес к исследованию и моделированию свойств мягких материалов [1,2]. Это в значительной мере определяется прогрессом в области синтеза новых полимерных композитных материалов с контролируемыми свойствами [3] и обнаружением новых магнитных наночастиц с анизометрией формы [4] и анизотропной внутренней структурой $[5,6]$. Для управления термодинамическими свойствами таких систем можно использовать как внешние (температура, электрические и магнитные свойства), так и внутренние (форма, структура, топология) параметры [7]. Новейшей отраслью в этой области являются системы с магниточувствительными компонентами [8]. Наиболее простым и распространенным полимерным композитом с магнитными свойствами являются магнитные частицы, покрытые полимерной оболочкой, которая устанавливает стерический барьер, препятствующий необратимой агломерации. Примерами таких систем являются магнитные феррожидкости $[9,10]$ или магнитореологические суспензии.

Следующими по сложности считаются магнитные композитные материалы, состоящие из нано- и микроразмерных магнитных частиц, внедренных в полимерную матрицу. Однако, их успешное применение и создание новых материалов на их основе невозможно без фундаментального понимания их микроструктуры, макроскопического поведения и реологических свойств. Все эти свойства в значительной мере определяются параметрами самих частиц: их формой и внутренней структурой. А также, сами по себе магнитные наночастицы имеют большие перспективы в биомедицинских приложениях [11-13]. С одной стороны, можно контролировать их размеры и структуру, чтобы они соответствовали различным биологическим объектам в природе. С другой стороны, магнитные наночастицы могут быть функционализированы молекулами для взаимодействия или соединения с биологическими объектами различных типов. Более того, можно эффективно управлять ими с помощью внешнего магнитного поля. Такая возможность открывает новые пути транспортировки или локализации лекарств, контрастных веществ и т.д. магнитными частицами, а также новые области применения.

Стоит отметить, что изучение микроструктуры систем с магнитными анизотропными наночастицами [14] по-прежнему актуально и представляет огромный интерес, несмотря на то, что исследования мягких материалов начались достаточно давно. Большинство работ в этой области являются экспериментальными; однако они являются дорогостоящими и не позволяют синтезировать частицы в достаточных объемах для изучения их различными методиками. Поэтому возникает необходимость в создании новых теоретических моделей и алгоритмов для компьютерных экспериментов, ведь 
это позволяет объяснить эксперименты и разработать новую стратегию синтеза усовершенствованных смартматериалов.

В настоящей работе мы не просто продолжаем исследование, начатое в работе [15], а расширяем его, следуя за синтезом частиц. В данный момент возможно создание магнитных наночастиц различной формы и с разной ориентацией магнитных моментов внутри частицы. Это открывает новые возможности для их применения благодаря разнообразию самоорганизации таких частиц, а значит, и их макросвойств. В рамках настоящей работы нами было проведено комплексное исследование системы магнитных анизотропных эллипсоидальных наночастиц, основанное на сочетании компьютерного моделирования и аналитических методов. Такой подход позволяет определить основные преимущества и недостатки высокотехнологичного использования этих частиц. В работе исследованы два случая ориентации диполя внутри каждой частицы: расположение параллельно главной оси вращения эллипсоида (никелевые наноиглы) или перпендикулярно ей (гематитовые наноэллипсоиды). Полученные результаты выявили влияние ориентации магнитного момента внутри частиц одинаковой формы на самоорганизацию системы, ее микроскопические и макроскопические свойства. Таким образом, форма и внутренняя структура частицы вместе позволяют сочетать разные свойства системы (агрегированность и магнитный отклик), что еще больше приближает нас к разработке новых материалов с точно контролируемыми свойствами.

\section{2. Описание системы}

В работе рассмотрена система, состоящая из магнитных анизотропных монодисперсных наночастиц. Магнитный момент $\mathbf{m}_{i}$ расположен в центре масс частицы $i$, частица имеет форму эллипсоида. Мы исследовали упрощенный эллипсоид вращения с различной ориентацией магнитного момента: параллельно или перпендикулярно главной оси. Его объем можно вычислить как $V=4 \pi a^{2} b / 3$, а соотношение полуосей задается параметром $X_{0}=b / a$, где $b$ - это главная ось эллипсоида. Мы рассматривали наночастицы только с показателем эллиптичности $X_{0}>1$ и выбранными значениями параметра $X_{0}=1.1,1.4,1.7,2.0,2.3$.

Для описания магнитного взаимодействия был использован потенциал $U_{d}$ :

$$
U_{d}(i, j)=-\left[3\left(\mathbf{m}_{i}, \mathbf{r}_{i j}\right) / r_{i j}^{5}-\left(\mathbf{m}_{i}, \mathbf{m}_{j}\right) / r_{i j}^{3}\right],
$$

где $\mathbf{r}_{i j}$ - радиус-вектор двух частиц, $\mathbf{m}_{i(j)}$ - магнитный момент частицы $i(j)$.

Для описания стерического взаимодействия анизотропных частиц использовался потенциал ГейБерне $[16,17]$, широко применяемый для исследования несферических частиц. Этот потенциал учитывает не только расстояние между центрами частиц, но и их ориентацию. Он не является центральным и зависит от взаимной ориентации частиц. Выражение для потенциала Гей-Берне имеет вид

$$
U_{G B}=\left\{\begin{array}{ll}
4 \varepsilon()\left[A^{12}()-A^{6}()\right]+\varepsilon(), & r_{i j} \leq r_{c} \\
0, & r_{i j}>r_{c}
\end{array},\right.
$$

где

$$
\begin{gathered}
\left.A()=A\left(\mathbf{u}_{i}, \mathbf{u}_{j}, \mathbf{R}_{i j}\right)=\sigma_{0} /\left(\mathbf{u}_{i}, \mathbf{u}_{j}, \mathbf{R}_{i j}\right)+\sigma_{0}\right) \\
\sigma\left(\mathbf{u}_{i}, \mathbf{u}_{j}, \mathbf{R}_{i j}\right)=\sigma_{0}\left[1-\varphi / 2\left(\mathbf{R}_{i j} \mathbf{u}_{i}+\mathbf{r}_{i j} \mathbf{u}_{j}\right)^{2} /\left(1+\varphi \mathbf{u}_{i} \mathbf{u}_{j}\right.\right. \\
+\left(\mathbf{R}_{i j} \mathbf{u}_{i}-\mathbf{r}_{i j} \mathbf{u}_{j}\right)^{2} /\left(\mathbf{R}_{i j} \mathbf{u}_{i}-\mathbf{r}_{i j} \mathbf{u}_{j}\right)^{2} /\left(1-\varphi \mathbf{u}_{i} \mathbf{u}_{j}\right]^{-1 / 2}, \\
\varepsilon() \equiv \varepsilon\left(\mathbf{u}_{i} \mathbf{u}_{j}\right)=\varepsilon_{0}\left[1-\varphi^{2}\left(\mathbf{u}_{i} \mathbf{u}_{j}\right)^{2}\right]^{-1 / 2}, \\
\varphi=\left[X_{0}^{2}-1\right]\left[X_{0}^{2}+1\right] .
\end{gathered}
$$

В данном случае за вектор $\mathbf{R}_{i j}=\mathbf{r}_{i j} / r_{i j}=\left(\mathbf{r}_{i}-\mathbf{r}_{j}\right) / r_{i j}$ принимается единичный вектор, коллинеарный вектору, соединяющему центры частиц, а $\mathbf{u}_{i(j)}$ - единичный вектор, направленный вдоль главной оси, $r_{c}-$ критический радиус, равный $r_{c}=\left(2^{1 / 6}-1\right) \sigma_{0}+\sigma\left(\mathbf{u}_{i}, \mathbf{u}_{j}, \mathbf{R}_{i j}\right)$. Значение параметра $\sigma_{0}$ принимается равным $\sigma_{0}=\sqrt{ } 2 D$, где $D$ - является диаметром частицы.

\section{3. Компьютерный эксперимент}

Для проведения комплексного анализа системы при различных параметрах, а также для изучения взаимодействия между частицами, были проведены компьютерные эксперименты (компьютерные симуляции). При моделировании за единицу измерения длины был взят диаметр эллипсоида в основном поперечном сечении (диаметр $D=2 \mathrm{~nm}$ ). Для исследования в широком диапазоне параметров была рассмотрена система, состоящая из 512 наночастиц; для расчета дальнодействующего магнитного диполь-дипольного взаимодействия использовался алгоритм $\mathrm{P}^{3} \mathrm{M}[18]$. Для достижения равновесного состояния был проведен начальный расчет с периодом $10^{5} \Delta t^{*}$, после чего было начато исследование микроструктуры и макроскопических характеристик системы. Для частиц различной формы проводилось исследование при одинаковой числовой концентрации $1 \rho$ в широком диапазоне значений. Во всех компьютерных экспериментах использовались периодические граничные условия, вычисления проводились в среде ESPResSo. В данных экспериментах использованы безразмерные параметры; все величины, связанные с длиной, нормированы на значение короткой оси эллипсоида (она все время равна 1). Также использовалась безразмерная температура $T^{*}=k T / \varepsilon_{0}=1$, где $k T$ - это тепловая энергия, а $\varepsilon_{0}-$ энергетический параметр. Безразмерный магнитный моменттm измерялся в единицах $1 / \sqrt{ } T^{*}$ и был расположен в центре частицы.

Использование безразмерных параметров позволяет изучать различные наночастицы, обладающие схожими 
свойствами. Примерами таких систем могут служить сферические магнитные наночастицы из магнетита (намагниченность насыщения которого $480 \mathrm{kA} / \mathrm{m}$ ), внедренные в эллипсоидальные немагнитные мягкие наночастицы. Для таких частиц безразмерный магнитный момент $\mathbf{m}^{2}=5$ при комнатной температуре соответствует частице с диаметром около $15 \mathrm{~nm}$, а для $\mathbf{m}^{2}=3$ диаметр равен $12.5 \mathrm{~nm}$. Также могут быть рассмотрены никелевые наноиглы (намагниченность насыщения материала $488 \mathrm{kA} / \mathrm{m}$ ), покрытые немагнитной оболочкой, с диаметром в центральном сечении порядка $50 \mathrm{~nm}$ и различной длиной (например, для $X_{0}=2.3$ равной $115 \mathrm{~nm}$ ). Использовался метод молекулярной динамики [19], компьютерное моделирование проводилось для случая комнатной температуры.

Из компьютерных экспериментов были получены большие массивы данных, содержащие информацию о координатах частиц и ориентации их магнитных моментов. На основании этих данных была изучена микроструктура системы и ее макроскопические свойства, а именно, была рассчитана начальная восприимчивость системы и проведен кластерный анализ.

\section{4. Макроструктура системы}

Прежде всего, мы проанализировали макроскопические параметры системы. Начальная магнитная восприимчивость является одной из важнейших характеристик магнитных мягких материалов, она эквивалентна наклону кривой намагничивания при нулевом поле. Это показывает, насколько сильны корреляции дипольных моментов в системе и насколько чувствительна система к внешнему бесконечно малому магнитному полю. Основой для анализа служат данные, полученные в результате компьютерного моделирования. Для расчета начальной восприимчивости использовалась следующая формула, основанная на флуктуационно-диссипационной теореме

$$
\chi=1 /\left(3 V \mu_{0} T^{*}\right) \cdot\left(\left\langle M^{2}\right\rangle-\langle M\rangle^{2}\right),
$$

где $M-$ суммарный магнитный момент системы и усреднение $\langle\ldots\rangle$ производится для всех статистически независимых конфигураций, $V-$ объем системы, $T^{*}-$ безразмерная температура.

Мы исследовали начальную восприимчивость при комнатной температуре для различных концентраций, значений магнитных моментов и соотношений полуосей эллипсоида. Ниже можно увидеть сравнение начальной восприимчивости для двух ориентаций магнитных моментов. Рис. 1, $a$ соответствует ориентации перпендикулярно главной оси, а рис. $1, b$ построен для параллельной ориентации, $\rho=0.04$. На всех графиках пунктирным линиям соответствуют значения параметра $X_{0}=1.1$, сплошным линиям $-X_{0}=1.7$, штриховым линиям $X_{0}=2.3$.
Из этих рисунков видно, что в случае параллельности главной оси начальная восприимчивость возрастает с увеличением магнитного момента. Подобное поведение мы наблюдали для различных концентраций. Также видно, что начальная восприимчивость частиц, форма которых близка к сферической, возрастает быстрее и достигает более высоких значений, чем в случае эллипсоидных частиц. Таким образом, с ростом анизотропии не только уменьшается абсолютное значение восприимчивости, но и его относительное изменение становится меньше. Общее уменьшение межчастичных дипольных корреляций/наблюдаемого магнитного отклика можно объяснить изменением конфигураций основного состояния и, следовательно, изменением микроструктуры системы.
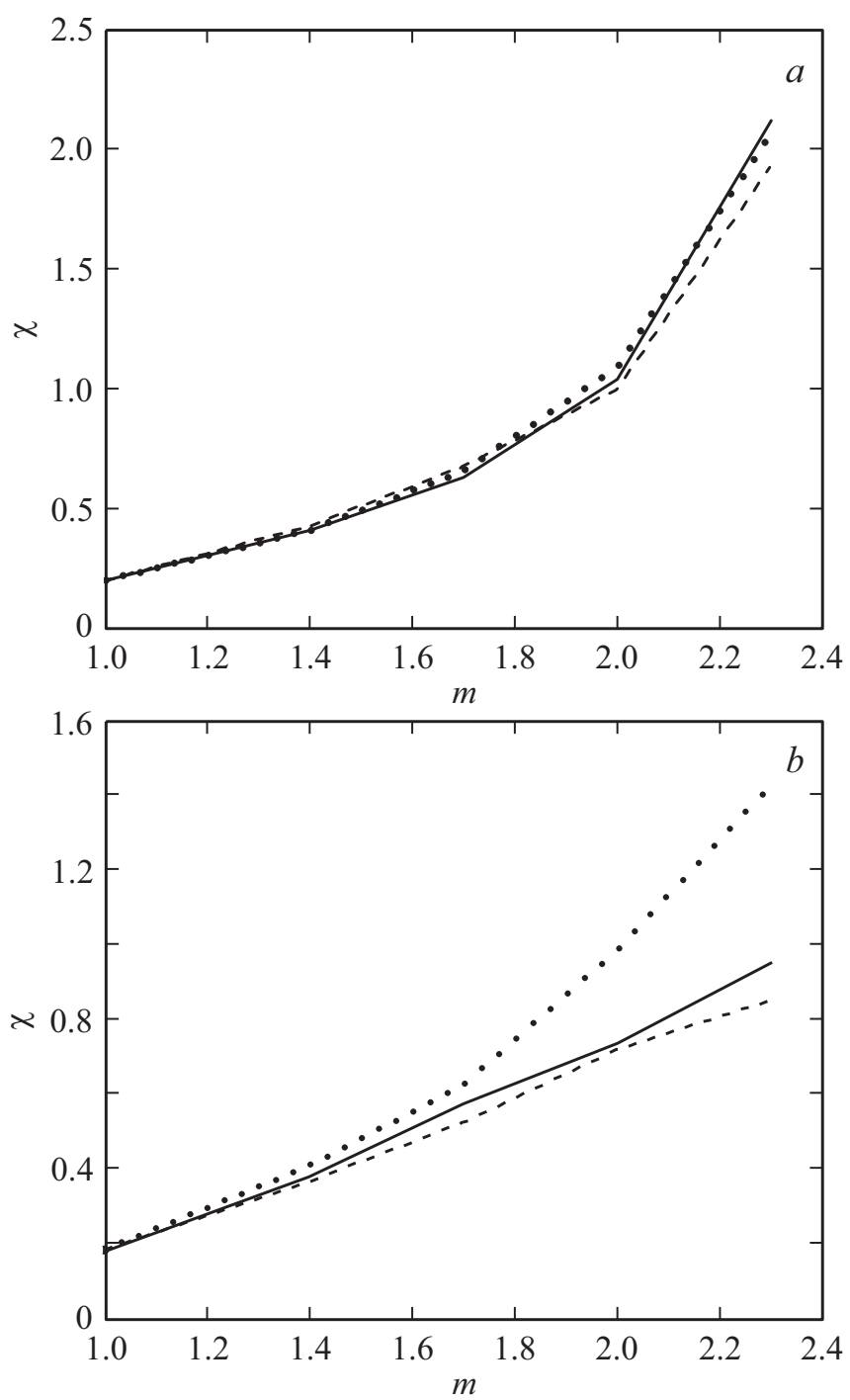

Рис. 1. Зависимость начальной восприимчивости от магнитных моментов наночастиц с концентрацией $\rho=0.04, X_{0}=1.1$ (пунктирные линии), $X_{0}=1.7$ (сплошные линии), $X_{0}=2.3$ (штриховые линии): $a-$ случай перпендикулярной ориентации магнитного момента, $b-$ случай параллельной ориентации магнитного момента. 
Для случая перпендикулярной ориентации величина магнитного момента не влияет на начальную восприимчивость так значительно, как в предыдущем случае. Увеличение магнитного момента приводит к росту начальной восприимчивости, тогда как форма практически никак не влияет на нее.

Анализ результатов, представленных на рис. 1, $a$ и $b$, позволяет утверждать, что начальная восприимчивость больше для системы с ориентацией диполей перпендикулярно главной оси. Такое поведение объясняется основным состоянием системы, которое является наиболее энергетически выгодной конфигурацией при $0 \mathrm{~K}$. Было показано, что для системы с магнитным моментом, направленным перпендикулярно оси вращения, форма частицы не влияет на структуру основного состояния, в отличие от второго случая, где явно существует переход. И это приводит к различной микроструктуре системы при комнатной температуре.

\section{5. Микроструктура системы}

Исследуем микроструктуру системы, чтобы получить более полное представление о поведении системы.

Для исследования микроструктуры системы проанализируем результаты кластерного анализа, который позволяет определить средние характеристики (длина кластера, заагрегированность) сформированных агрегатов в системе. Средняя длина кластера показывает, сколько частиц находится в кластерах, а заагрегированность определяет долю всех частиц, находящихся в кластерах. Критерием отбора частиц в кластеры является следующее: энергия магнитного взаимодействия должна быть отрицательной, а межчастичное расстояние не должно превышать критическое. Табл. 1 показывает средние характеристики системы с магнитными моментами, направленными параллельно главной оси, в зависимости от магнитного момента и формы наночастицы. Последняя строка таблицы соответствует проценту конфигураций магнитных моментов „голова-хвост“. Для этого примера концентрация частиц равна $\rho=0.04$ :

На основании этой таблицы можно сделать вывод о том, что наночастицы, форма которых близка к сферической, в отличие от вытянутых эллипсоидальных частиц, демонстрируют значительно более сильное кластерообразование, причем увеличивается не только число кластеров в системе, но и среднее число частиц в кластере. Увеличение магнитного момента в случае почти сферических частиц приводит к увеличению числа агрегатов в системе. Однако такого поведения не наблюдается для вытянутых частиц, система остается почти изотропной. Такая разница в микроструктуре приводит к различию в поведении начальной восприимчивости. Кроме того, анализ взаимной ориентации показывает, что для почти сферических частиц преобладает ориентация „голова-хвост“, что сказывается на росте начальной восприимчивости. Наличие таких конфигураций позволяет
Таблица 1. Средние характеристики системы с магнитными моментами, направленными перпендикулярно главной оси, при $\rho=0.04$

\begin{tabular}{l|c|c|c|c}
\hline \multicolumn{4}{c|}{ Концентрация $\rho=0.04$} \\
\hline \multicolumn{1}{c|}{\begin{tabular}{c} 
Соотношение полуосей \\
\multicolumn{1}{c|}{ эллипсоида $X_{0}$}
\end{tabular}} & \multicolumn{2}{|c|}{1.1} & \multicolumn{2}{c}{2.3} \\
\hline Магнитный момент $\mathbf{m}^{2}$ & 1 & 5 & 1 & 5 \\
Средняя длина кластера $L$ & 2.062 & 3.112 & 1.974 & 2.037 \\
Заагрегированность системы $P$ & 7.3 & 61.22 & 1.577 & 6.956 \\
Взаимная ориентация & $37 \%$ & $71 \%$ & $4 \%$ & $1 \%$ \\
диполей в кластере & & & &
\end{tabular}

Таблица 2. Средние характеристики системы с магнитными моментами, направленными перпендикулярно главной оси, при $\rho=0.04$

\begin{tabular}{l|c|c|c|c}
\hline \multicolumn{4}{c|}{ Концентрация $\rho=0.04$} \\
\hline \multicolumn{1}{c|}{\begin{tabular}{c} 
Соотношение полуосей \\
\multicolumn{1}{c|}{ эллипсоида $X_{0}$}
\end{tabular}} & \multicolumn{2}{|c|}{1.1} & \multicolumn{2}{c}{2.3} \\
\hline Магнитный момент $\mathbf{m}^{2}$ & 1 & 5 & 1 & 5 \\
Средняя длина кластера $L$ & 2.07 & 5.282 & 2.0003 & 3.326 \\
Заагрегированность системы $P$ & 7.85 & 84.969 & 2.314 & 64.644 \\
Взаимная ориентация & $50 \%$ & $93 \%$ & $51 \%$ & $96 \%$ \\
диполей в кластере & & & &
\end{tabular}

системе сильнее реагировать на внешнее поле, в отличие от системы со случайными ориентациями магнитных моментов. Но для вытянутых наночастиц преобладает антипараллельная взаимная ориентация. Таким образом, магнитный момент практически не влияет на поведение системы с эллипсоидальными частицами, магнитные моменты которых сонаправлены оси вращения, поэтому она остается изотропной. Это отражается и на поведении начальной восприимчивости, которая в этом случае практически совпадает с начальной восприимчивостью системы с изотропным распределением частиц без кластеров.

В табл. 2 представлены средние характеристики системы с перпендикулярными главной оси магнитными моментами, в зависимости от магнитного момента и формы наночастицы. Последняя строка в таблице соответствует проценту конфигураций „голова-хвост“. Для этого примера концентрация также равна $\rho=0.04$.

В этом случае можно сделать вывод о том, что общее количество кластеров в системе увеличивается, но среднее число частиц в кластере существенно не меняется. Увеличение магнитного момента в случае почти сферических частиц приводит к увеличению числа агрегатов в системе. Аналогичная зависимость наблюдается и в случае вытянутых частиц. В обоих случаях преобладает ориентация „голова-хвост ${ }^{6 .}$. Таким образом, форма частиц с таким типом ориентации диполя не оказывает значительного влияния на поведение системы. 


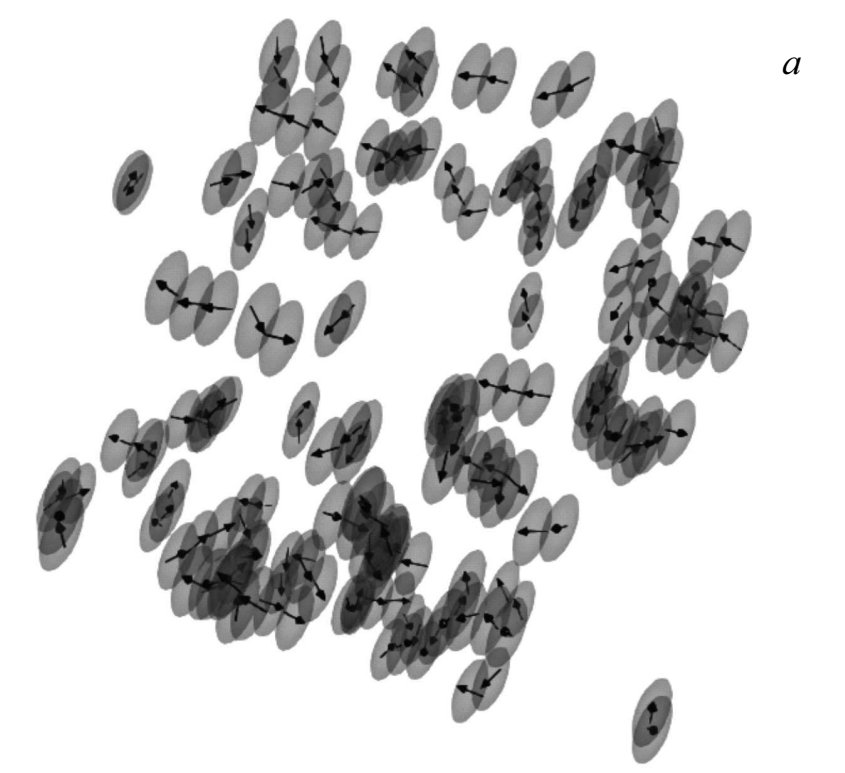

$b$
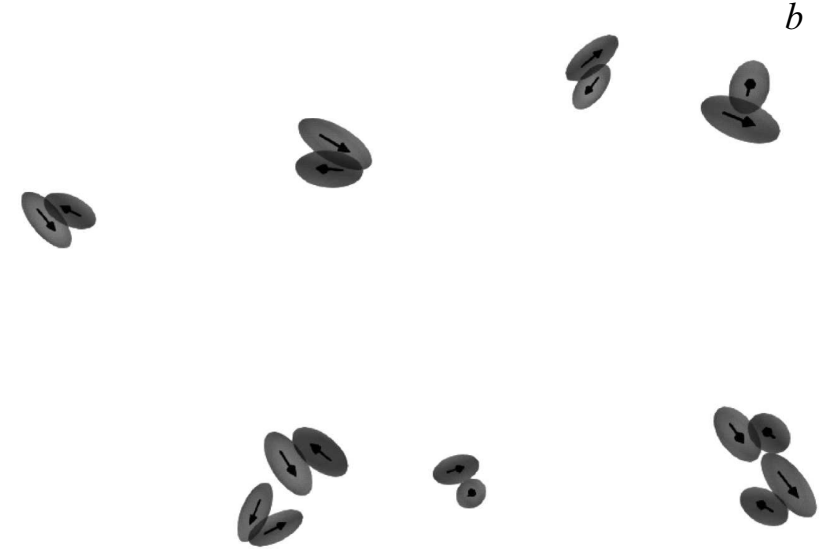

Рис. 2. Наглядное представление кластеров системы с концентрацией $\rho=0.04$, параметрами $X_{0}=2.3$ и $m^{2}=5: a-$ случай перпендикулярной ориентации магнитного момента, $b-$ случай параллельной ориентации магнитного момента.

Проведенный анализ позволяет сделать следующие выводы. Средняя длина кластера и заагрегированность системы больше для частиц с ориентацией магнитного момента перпендикулярно оси вращения. С ростом магнитного момента для почти сферических частиц в системе увеличивается количество конфигураций „головахвост“. Подавляющее большинство конфигураций наблюдается при ориентации магнитного момента перпендикулярно оси вращения. Тенденция сохраняется и в случае системы с эллипсоидальными частицами и той же ориентацией. А в системе эллипсоидальных частиц с магнитным моментом вдоль главной оси подавляющее большинство составляют антипараллельные пары.

Более наглядное представление о системе эллипсоидальных частиц приведено на рис. 2, $a$ и $b$. При этом изображены только кластеры, одиночные частицы не показаны. На рис. 2, а представлена система частиц с магнитными моментами, перпендикулярными оси вращения, на рис. 2, $b$ - параллельными оси вращения. Для этого примера выбрана концентрация $\rho=0.04$.

\section{6. Заключение}

Создание и использование магнитных композитных материалов невозможно без фундаментального понимания их микроструктуры, макроскопического поведения и реологических свойств. И даже самый простой пример таких материалов - суспензии отдельных магнитных частиц - при изменении их формы и внутренней структуры могут служить для различных промышленных и медико-биологических приложений. В настоящей работе мы изучили микроструктуру и магнитные свойства эллипсоидальных наночастиц с различной ориентацией диполя: параллельно и перпендикулярно главной оси вращения.

Первой была исследована начальная восприимчивость системы - ее отклик на бесконечно слабое внешнее магнитное поле. Для случая параллельной главной оси ориентации видно, что начальная восприимчивость частиц, форма которых близка к сферической, быстрее возрастает и достигает более высоких значений, чем в случае эллипсоидных частиц. Таким образом, с ростом анизотропии формы уменьшается не только абсолютное значение восприимчивости, но и ее относительное изменение. Перпендикулярная ориентация магнитного момента не влияет на начальную восприимчивость так существенно, как в предыдущем случае. Увеличение магнитного момента приводит к росту начальной восприимчивости, тогда как форма частицы практически не оказывает на нее влияния. Для более точного понимания причины такого поведения был проведен кластерный анализ. Показано, что средняя длина кластера и заагрегированность системы больше для частиц с ориентацией магнитного момента перпендикулярно оси вращения. Было показано, что в зависимости от ориентации магнитного момента и формы частицы, в системе преобладают кластеры разных типов с разной ориентацией магнитных моментов внутри него, что согласуется с основным состоянием системы, а также влияет на макроскопическое поведение системы. Для перпендикулярной оси вращения ориентации система подобна системе сферических частиц, а для параллельной оси вращения ориентации происходит структурный переход, магнитное взаимодействие ослабляется стерическим.

С ростом магнитного момента для близких к сферическим частиц в системе растет количество конфигураций „голова-хвост“. При ориентации магнитного момента перпендикулярно оси вращения тенденция сохраняется и в случае системы с эллипсоидальными частицами. А в системе эллипсоидальных частиц с магнитным моментом, параллельным главной оси, подавляющее большинство составляют антипараллельные пары. Все это (изменение размеров кластеров и ориентации маг- 
нитных моментов внутри кластера) объясняет поведение начальной восприимчивости. Таким образом, анизотропия формы и внутренняя структура частицы существенно влияет на микроструктуру системы магнитных частиц при комнатной температуре и на ее макроскопические свойства. Это может оказаться очень важным фактором при использования подобных наночастиц в медицине, когда сильный магнитный отклик наночастиц должен сочетаться с отсутствием значительного кластерообразования. Такие системы могут полностью воплощать идею настройки и разработки новых материалов с контролируемыми свойствами.

\section{Финансирование работы}

Данное исследование выполнено при финансовой поддержке гранта РНФ 19-72-10033.

\section{Конфликт интересов}

Авторы заявляют, что у них нет конфликта интересов.

\section{Список литературы}

[1] A. Tokarev, J. Yatvin, O. Trotsenko, J. Locklin, S. Minko. Adv. Functional Mater. 26, 22, 3761 (2016).

[2] J. Phillips. Phys. Today 73, 11 (2020).

[3] M. Nakade, T. Ikeda, M. Ogawa. J. Mater. Sci. 42, 4815 (2007).

[4] L. Rossi, S. Sacanna, W. Irvine, P. Chaikin, D. Pine, A. Philipse. Soft Matter 7, 9, 4139 (2011).

[5] L. Baraban, D. Makarov, M. Albrecht, N. Rivier, P. Leiderer, A. Erbe. Phys. Rev. E 77, 031407 (2008).

[6] S. Smoukov, S. Gangwal, M. Marquez, O.D. Velev. Soft Matter 5, 1285 (2009).

[7] R.V. Ulijn. J. Mater. Chem. 16, 2217 (2006).

[8] G. Filipcsei, I. Csetneki, A. Szilagyi, M. Zrinyi. Adv. Polymer Sci. 206, 1, 137 (2006).

[9] G. Rickayzen. Mol. Phys. 98, 10, 683 (2000).

[10] I. Torres-Diaz, C. Rinaldi. Soft Matter 10, 8584 (2014).

[11] Q.A. Pankhurst, N.T.K. Thanh, S.K. Jones, J. Dobson. J. Phys. D: Appl. Phys. 42, 22, 224001 (2009).

[12] L. LaConte, N. Nitin, G. Bao. Mater. Today 8, 5, 32 (2005).

[13] G.V. Kurlyandskaya, I. Novoselova, V.V. Schupletsova, R. Andrade, N.A. Dunec, L. Litvinova, A.P. Safronov, K.A. Yurova, N. Kulesh, A.N. Dzyuman, I. Khlusov. JMMM 431, 249 (2017).

[14] D. Lisjak, A. Mertelj. Progress. Mater. Sci. 95, 286 (2018).

[15] А.В. Гудкова, Е.С. Пьянзина. ФТТ 59, 11, 2159 (2017).

[16] J.G. Gay, B.J. Berne. J. Chem. Phys. 74, 3316 (1981).

[17] S. Kantorovich, E. Pyanzina, C. De Michele, F. Sciortino. Soft Matter 9, 4412 (2013).

[18] A. Arnold, B.A. Mann, H. Limbach, C. Holm. Gesellschaft für wissenschaftliche Datenverarbeitung mbH 63, 43 (2004).

[19] Z. Wang, C. Holm, H.W. Muller. Phys. Rev. E 66, 2, 021405 (2002).

Редактор Е.В. Толстякова 